\title{
Giant Magnetorheological Effect of Agar Magnetic Gels
}

\author{
Tetsu Mitsumata, Takafumi Yamamoto, Emiko Suzuki, and Jun-ichi Takimoto \\ Department of Polymer Science and Engineering, Faculty of Engineering, Yamagata University, Yonezawa 992-8510, Japan
}

Fax: 81-238-26-3101, e-mail: tetsu(a)yz.yamagata-u.ac.jp

Effects of matrix on the giant reduction in storage modulus of magnetic gels have been investigated using agar and carrageenan gels. The complex modulus was measured before and after magnetization of the gel by dynamic viscoelastic measurements with a compressional strain. The agar magnetic gels showed a large nonlinear viscoelastic response and magnetically-induced deformation. The giant reduction in the storage modulus of $\sim 10^{5}$ $\mathrm{Pa}$, and also in the loss modulus of $\sim 10^{4} \mathrm{~Pa}$ was observed for agar magnetic gels, as well as carrageenan one. The deformation due to magnetization seen in agar magnetic gels was larger than that in carrageenan by more than twice. Rheological data, deformation due to magnetization, and morphologies of magnetic particles have been discussed.

Key words: gels, magnetic gel, modulus, rheology, agar

\section{INTRODUCTION}

The polymer gels containing magnetic fluids respond to magnetic fields and it is called as magneto-rheological or magneto-sensitive gels [1]. Compressive modulus of the magnetic gel with the matrix of a poly(vinyl alcohol) (PVA) swollen by a magnetic fluid, increased under uniform magnetic fields [2]. The modulus change occurred simultaneously with an application of the magnetic field; it increased with increasing magnetic field strength and saturated above $2 \mathrm{kOe}$. It was considered that the modulus change was brought by the strong coupling between polymer networks and magnetic fluids. The change in the modulus from 0 to 4 $\mathrm{kOe}$ was $31 \mathrm{~Pa}$, which equals to $19 \%$ increases of that without the field. Magnetic gels loaded with magnetic fine particles also show the magnetorheological effect. Chemically cross-linked PVA gels containing magnetic fine particles undergo changes in longitudinal modulus at $10 \mathrm{MHz}$ and in dynamic modulus at $10^{-1} \mathrm{~Hz}-10^{2} \mathrm{~Hz}$ due to magnetization [3-6].

Recently, we reported that $\kappa$-carrageenan magnetic gel demonstrates the giant modulus reduction due to magnetization $[7,8]$. The magnetic gel contains barium ferrite which is a kind of ferrimagnetic particles. The complex modulus was measured before and after magnetization of the gel by a dynamic viscoelastic apparatus with a compressional strain mode. The gels showed giant reduction in the storage modulus $\sim 10^{7} \mathrm{~Pa}$, and also in the loss modulus $\sim 10^{6} \mathrm{~Pa}$ due to magnetization. The reduction increased with increasing the volume fraction of the ferrite, and it was nearly independent of the frequency. It was also found that the change in the modulus was nearly independent of a magnetization direction and irradiation time of magnetic fields to the gel. The magnetic gels demonstrating the giant reduction in the dynamic modulus showed a large nonlinear viscoelastic response. It was observed that the magnetic gel was slightly deformed due to magnetization. The observed giant complex modulus reduction could be attributed to the nonlinear viscoelasticity and deformation caused by magnetization.
In this paper, we have investigated the effects of matrix properties on the modulus change using agar magnetic gels in order to understand the giant magnetorheological effect seen in $k$-carrageenan magnetic gels. Rheological and morphological data were presented and discussed in this paper.

\section{EXPERIMENTAL PROCEDURES}

\subsection{Synthesis of Magnetic Gel}

Magnetic gels, which consist of a finely dispersed powder of barium ferrite and agar of a natural polymer, have been synthesized. A pre-gel solution of the magnetic gel was prepared by mixing the $5 \mathrm{wt} \%$ agar (Wako Chemicals) aqueous solution and the barium ferrite $\mathrm{BaFe}_{12} \mathrm{O}_{19}$ (Sigma-Aldrich Co.) at $90{ }^{\circ} \mathrm{C}$. The pre-gel solution was cooled down to $20^{\circ} \mathrm{C}$, and the magnetic gel was obtained. The mean diameter of a magnetic particle was determined as $15 \mu \mathrm{m}$. The diameter of barium ferrite is much larger compared to magnetic fluid, so it enables the gel to have a remanent magnetization even though the magnetic field is not applied. The weight ratio of ferrite to that of agar $c_{f}$ was varied up to $60 \mathrm{wt} \%$ which corresponds to the volume fraction of $22 \mathrm{vol} . \%$. To find out magnetization effects, the gel was put under a uniform magnetic field of $1 \mathrm{~T}$ in order to give the gel a remanent magnetization.

\subsection{Rheological Measurements}

Dynamic viscoelastic measurements were carried out using a Dve Rheospectora (Dve-V4, Rheology Co., Ltd). Oscillatory compressional shear $\gamma=\gamma_{0} \sin 2 \pi f t$ was applied to magnetic gels and the stress response was measured; hence elastic modulus obtained stands for Young's modulus. The frequency range was varied from $10^{-1} \mathrm{~Hz}$ to $10^{2} \mathrm{~Hz}$, and the oscillation amplitude was varied from $1 \times 10^{-5}$ to $9 \times 10^{-3}$. The offset strain with respect to the sample thickness was kept as $3 \%$. The temperature was maintained at $20.0 \pm 1.0{ }^{\circ} \mathrm{C}$ during the viscoelastic measurement. The shape of samples for the mechanical measurement was cubic with dimensions of approximately $10 \times 10 \times 10 \mathrm{~mm}$. Each modulus was 
determined from an average of three measurements.

\subsection{Magnetic Measurements}

Magnetization measurements up to $1 \mathrm{~T}$ were carried out by a vibrating sample magnetometer (VSM-P7, Toei Industrial Co., Ltd.). Shape of the sample is a disk with $2 \mathrm{~mm}$ in diameter and $1 \mathrm{~mm}$ in thickness. The magnetic gel was wrapped in a thin film of poly(vinyl chloride) to avoid evaporating water from the gel. Magnetization of the magnetic gel was calibrated by the disk-shaped $\mathrm{Ni}$ in order to eliminate the demagnetizing effect.

\subsection{Measurements of deformation}

Dimension that is perpendicular to the magnetization was measured using a laser displacement sensor with the maximum resolution of $0.1 \mu \mathrm{m}$ (ZS-HLDS5, Omron Co.). We measured the dimensions of a cubic shaped magnetic gel with the size of $10 \times 10 \times 10 \mathrm{~mm}$ before and after magnetizing at $1 \mathrm{~T}$.

\subsection{Microscope Observations}

Microscope observations for agar and carrageenan magnetic gels were carried out using an upright microscope (Axio Imager Mlm, Carl Zeiss, Inc.). The film-shaped gel with approximately $10 \mu \mathrm{m}$ in thick was observed by transmission light.

\section{RESULTS AND DISCUSSIONS}

Fig. 1 shows photographs of the magnetic particles dispersed in carrageenan and agar magnetic gels. As seen in the photograph, dispersibility of magnetic particles strongly depends on the kind of matrix. The diameter for large particles for agar and carrageenan gels was $\sim 100 \mu \mathrm{m}$ and $\sim 200 \mu \mathrm{m}$, respectively. According to the micrographs obtained by a scanning electron microscopy, the mean diameter of the magnetic particle
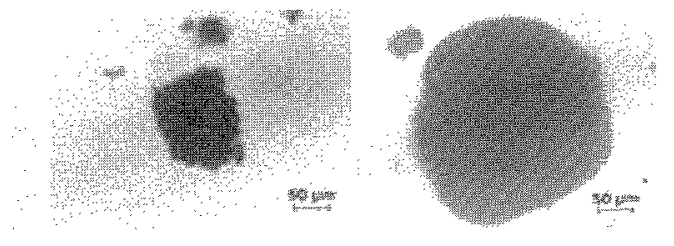

Fig. 1. Microphotographs of the magnetic particles dispersed in agar (left) and carrageenan (right) gels.

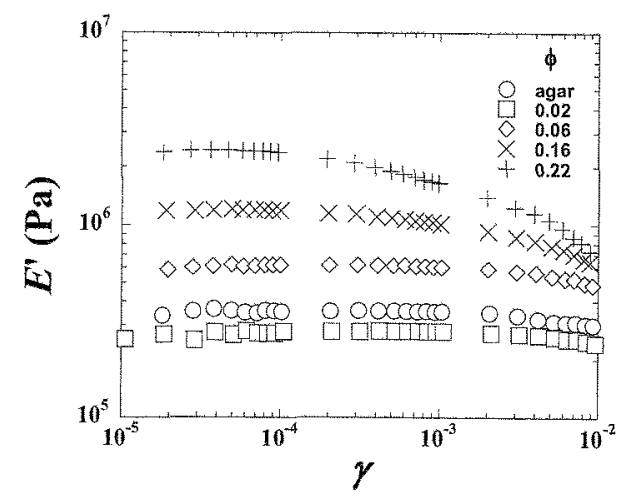

Fig. 2. Strain dependence of the storage modulus at $1 \mathrm{~Hz}$ for agar magnetic gel with various volume fractions. was distributed around few microns. This suggests that the magnetic particle forms secondary particles both in agar and carrageenan gels. It has a trend that the magnetic particles in carrageenan gels make secondary particles more easily than agar gels. The maximum volume fraction of magnetic particles was 0.37 and 0.22 for carrageenan and agar gels, respectively.

Fig. 2 shows the strain dependence of the storage modulus at $1 \mathrm{~Hz}$ for agar magnetic gels with various volume fractions of magnetic particle. The storage modulus of an agar gel without magnetic particles lied approximately $\sim 10^{5} \mathrm{~Pa}$; the modulus was insensitive to strain at all strains. In contrast, the storage modulus of agar magnetic gels was constant only below $\gamma \sim 3 \times 10^{-4}$ and it decreased largely with strain indicating nonlinear viscoelastic response. This means that the magnetic particles in the magnetic gel form a weak structure, which is easy to break at very small strains.

Fig. 3 shows the storage modulus of the magnetic gels as a function of the volume fraction of magnetic particles. The storage modulus was normalized by those of matrices, i.e. agar or $\kappa$-carrageenan. The storage modulus largely increased with the volume fraction, and the modulus at $\phi=0.22$ was higher than that of an agar gel without particles by 8 times. The solid line in the figure represents the modulus $E^{\prime}$ calculated by

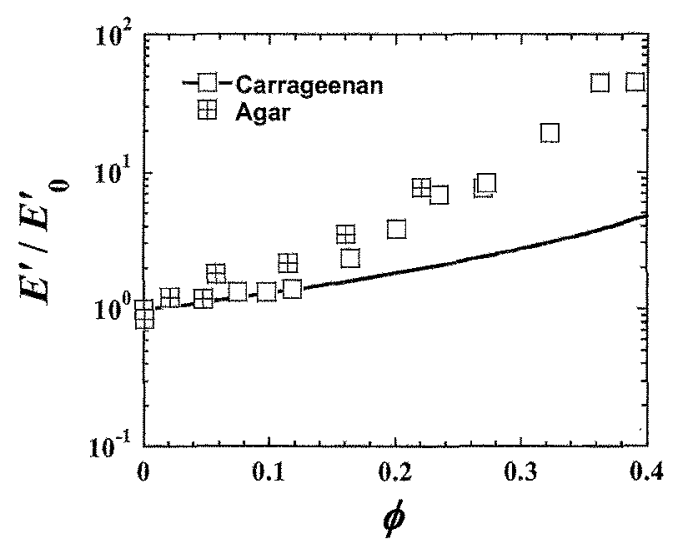

Fig. 3. Volume fraction dependence of the storage modulus at $1 \mathrm{~Hz}$ for agar and carrageenan magnetic gels.

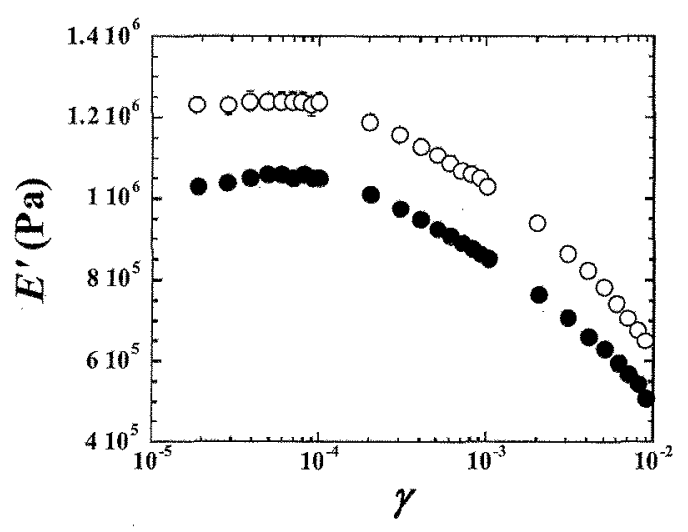

Fig. 4. Strain dependence of the storage modulus at $1 \mathrm{~Hz}$ for agar magnetic gel before and after magnetization at $1 T$ $(\phi=0.16)$. 
Krieger-Dougherty equation as following [9]

$$
E^{\prime}=E_{0}^{\prime}\left(1-\frac{\phi}{\phi_{m}}\right)^{-\frac{5}{2} \phi_{m}}
$$

where $E^{\prime}$ and $E_{0}^{\prime}$ show the storage modulus of the magnetic gel and matrices, respectively. $\phi_{\mathrm{m}}$ stands for the maximum volume fraction of magnetic particles, which nearly equals to be 0.63 for hard spheres [10]. The storage modulus below the volume fraction with 0.05 obeyed the above equation. This means that magnetic particles are randomly dispersed in the magnetic gel. The storage modulus above the volume fraction with 0.06 did not follow the equation suggesting magnetic particles are not randomly dispersed in the gel. It is considered that magnetic particles come in contact with each other and make a structure having high modulus. This consideration coincides with the data shown in Fig. 2: nonlinear viscoelastic response was very weak $\phi<0.05$ and was suddenly enhanced $\phi>0.06$.

Fig. 4 shows the effect of magnetization on the storage modulus for the agar magnetic gel with $\phi=0.16$. It was seen that the storage modulus after magnetization was lower than that before magnetization. The modulus decreased from $\sim 1.2 \times 10^{6} \mathrm{~Pa}$ to $\sim 1.0 \times 10^{6} \mathrm{~Pa}$, which corresponds to $1 / 6$ of the initial modulus before magnetization. This phenomenon was firstly observed in carrageenan magnetic gels that the storage modulus decreases more than $10^{7} \mathrm{~Pa}$ by magnetization [7]. This anomalous response is opposite to that of magnetic-responsive materials which have been reported in the past. For example, the compressive modulus of a magnetic gel consisting of PVA and magnetic fluids increased under magnetic field [2]. Silicone elastomers containing magnetic particles that were cured under magnetic fields also show the increase in storage moduli by applying magnetic fields [11]. Therefore, elastic moduli for these materials increase by applying magnetic fields.

To understand the mechanism of the giant modulus change observed we have theoretically estimated the change in the storage modulus due to magnetization. The magnetic energy is originated from a pair of magnetic momentum, and the energy density $U$ is

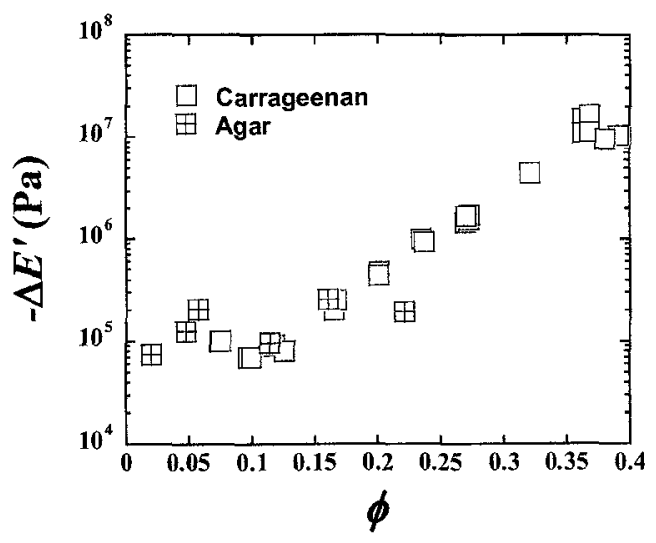

Fig. 5. Change in the storage modulus due to magnetization for agar and carrageenan magnetic gels as a function of volume fractions. proportional to $(M \phi)^{2} ; \quad M$ and $\phi$ stand for the magnetization and the volume fraction of magnetic particles. According to the dimension analysis, the change in the modulus should correspond to the energy density $U$, that is

$$
\Delta E^{\prime} \sim \mu_{0}(M \phi)^{2}
$$

When the volume fraction of magnetic particles was 0.22 , the magnetization was determined as $4 \times 10^{4} \mathrm{~A} / \mathrm{m}$ by magnetic measurements. Hence, the change in the storage modulus was estimated as $\sim 10^{2} \mathrm{~Pa}$. This value is three orders of magnitude lower than the observed modulus change of $\sim 10^{5} \mathrm{~Pa}$. This strongly suggests that the observed modulus change cannot be explained by only magnetic interactions between dispersed magnetic particles.

Fig. 5 shows the reduction in the storage modulus due to magnetization as a function of the volume fraction of magnetic particles. Data of the reduction measured for carrageenan gels were also presented in the figure. Agar magnetic gels showed nearly the same level of the reduction with carrageenan magnetic gels. The reduction for both gels was merely independent of the volume fraction below 0.12 ; it exponentially increased above the volume fraction. It has to mention that agar magnetic gels demonstrated a large reduction of $8 \times 10^{4} \mathrm{~Pa}$ even in lower volume fractions such as $\phi<0.06$. As described in Fig. 1, the sizes of secondary magnetic particles in agar were larger than that in carrageenan. It might be that the secondary particles contact with each other in a lower volume fractions.

Carrageenan magnetic gels were reported to undergo deformations due to magnetization [8]. Fig. 6 shows the deformation caused by magnetization for agar and carrageenan magnetic gels. Agar magnetic gels also demonstrated a considerable change in the deformation as seen in the figure. The deformation for both gels proportionally increased with the magnetic field. The deformation seen in agar magnetic gels was larger than that in carrageenan by more than twice. This might be originated from dispersibility of magnetic particles in the gels. As seen in Fig. 1, the diameter of magnetic particles in agar was smaller than that in carrageenan. Accordingly, magnetic particles in agar gel were

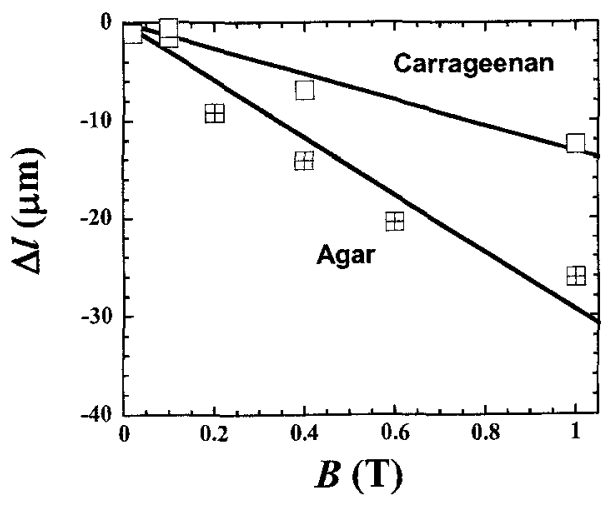

Fig. 6. Magnetic field dependence of the deformation induced by magnetization at $1 \mathrm{~T}$ for agar and carrageenan magnetic gels $(\phi \sim 0.16)$. 
randomly dispersed in the gel contrasting with carrageenan gels. Gels in which magnetic particles are well dispersed should be able to change its shape largely. It is because that the only part that can be deformed due to magnetization is gel network rather than magnetic particle. The deformation at the magnetic filed of $1 \mathrm{~T}$ reached $-26 \mu \mathrm{m}$, which corresponds to the strain with approximately $3 \times 10^{-3}$. According to Fig. 2, the storage modulus of the gel with $\phi=0.16$ at $3 \times 10^{-3}$ was $\sim 9 \times 10^{5} \mathrm{~Pa}$. The decrease in the storage modulus from the initial one was $\sim 2 \times 10^{5} \mathrm{~Pa}$. Giant reduction in the storage modulus can be explained by the Payne effect that is a rheological feature of magnetic gels, and a deformation induced by magnetization.

\section{CONCLUSION}

Effects of matrix on the giant reduction in the dynamic modulus have been investigated using agar and carrageenan matrices. Agar magnetic gels showed a large nonlinear viscoelastic response and underwent a magnetically-induced deformation, which are the features of carrageenan magnetic gels showing giant reduction in the storage modulus due to magnetization. Similar phenomenon was observed also in agar magnetic gels however the decrease in the modulus $\sim 10^{5} \mathrm{~Pa}$ was relatively small compared to that of carrageenan magnetic gels. Morphological studies revealed that the magnetic particle with few microns forms secondary particles both in agar and carrageenan gels. Magnetic particles in agar gel were randomly dispersed in the gel contrasting with carrageenan gels. Particles dispersion would play an important role to induce the giant reduction in the dynamic modulus for magnetic gels.

\section{Acknowledgements}

This research is partially supported by a Grant-in-Aid for Encouragement of Young Scientist from Japan Society for the Promotion of Science (Proposal No. 18750184), and Grant-in-Aid for Scientific Research of Priority Areas 438 from 2004-2008 Next - Generation Actuators Leading Breakthroughs.

\section{References}

[1] For example, M. Zrinyi, "Polymer Gels and Networks", Ed. by Y. Osada and A. R. Khokhlov, Marcel Dekker (2002) pp. 309-55.

[2] T. Mitsumata, K. Ikeda, J. P. Gong, Y. Osada, D. Szabo, and M. Zrinyi, J. App. Phys. 85, 8451-55 (1999).

[3] T. Mitsumata, E. Juliac, K. Furukawa, K. Iwakura, T. Taniguchi, and K. Koyama, Macromol. Rapid Commun. 23, 175-78 (2002).

[4] T. Mitsumata, K. Furukawa, E. Juliac, K. Iwakura, and K. Koyama, Int. J. Modern Phys. B 16, 2419-25 (2002).

[5] E. Juliac, T. Mitsumata, T. Taniguchi, K. Iwakura, and K. Koyama, J. Phys. Chem. B 107, 5426-31 (2003).

[6] T. Mitsumata, A. Nagata, K. Sakai, and T. Taniguchi, Jpn. J. Appl. Phys. 43, 8203-07 (2004).

[7] T. Mitsumata, A. Nagata, K. Sakai, and J. Takimoto, Macromol. Rapid Commun. 26, 1538-41 (2005).

[8] T. Mitsumata, K. Sakai, and J. Takimoto, J. Phys
Chem. 110, 20217-23 (2006).

[9] IM. Krieger and TJ. Dougherty, Trans. Soc. Rheol. 3, 137 (1959).

[10] GY. Onoda and ER. Liniger, Phys. Rev. Lett., 64, 2727 (1990).

[11] T. Shiga, A. Okada, and T. Kurauchi, J. Appl. Polym. Sci., 58, 787-92 (1995).

(Received December 8, 2006;Accepted March 1, 2007) 